九州大学学術情報リポジトリ

Kyushu University Institutional Repository

\title{
TESTS OF HOMOGENEITY FOR ORDERED ALTERNATIVES IN THE NORMAL POPULATIONS
}

Shirahata, Shingo

Department of Statistics, Faculty of General Education, Osaka University

https://doi.org/10.5109/13131

出版情報: 統計数理研究. 18 (3/4)，pp.61-68，1979-03. Research Association of Statistical Sciences

バージョン :

権利関係 : 


\title{
TESTS OF HOMOGENEITY FOR ORDERED ALTERNA- TIVES IN THE NORMAL POPULATIONS
}

\author{
By
}

\author{
Shingo Shirahata* \\ (Received March 27, 1978)
}

\begin{abstract}
Four new statistics to test the homogeneity of means and of variances against the ordered alternatives are considered and their power properties are investigated with the aid of computer simulations. These statistics are extensions of a partial ordering on the sample space induced by the likelihood ratio. It is found that they are more powerful than the likelihood ratio tests.
\end{abstract}

\section{Introduction.}

Let $X_{i j}$ for $j=1, \cdots, n_{i}$ and $i=1, \cdots, k$ be independent, normally distributed random variables with $E\left(X_{i j}\right)=\theta_{i}$ and $\operatorname{var}\left(X_{i j}\right)=\sigma_{i}^{2}$. We shall consider the following two problems. The one is to test the null hypothesis $H_{1} ; \theta_{1}=\cdots=\theta_{k}$ against the ordered alternative $K_{1} ; \theta_{1} \geqq \cdots \geqq \theta_{k}$ where at least one inequality is strict, under the constraint that $\sigma_{1}^{2}=\cdots=\sigma_{k}^{2}=\sigma^{2}$ and $\sigma^{2}$ is unknown. The other is to test the null hypothesis $H_{2} ; \sigma_{1}^{2}=\cdots=\sigma_{k}^{2}$ against the ordered alternative $K_{2} ; \sigma_{1}^{2} \geqq \cdots \geqq \sigma_{k}^{2}$ where at least one inequality is strict. If $\sigma^{2}$ is assumed to be known, the testing problem $\left(H_{1}, K_{1}\right)$ is included in Shirahata [15].

The method to approach to the problems is as follows. Suppose $\boldsymbol{X}$ be a random variable with a density function $f(\boldsymbol{x} ; \theta)$ where $\boldsymbol{x} \in R^{p}$ and $\boldsymbol{\theta} \in R^{q}$ for some $p$ and $q$ and consider the problem of testing $H_{0} ; \boldsymbol{\theta} \in \theta_{H_{0}}$ against the alternative $K_{0} ; \boldsymbol{\theta} \in \theta_{K_{0}}$. Let $\boldsymbol{x}$ and $\boldsymbol{y}$ be two elements of the sample space of $\boldsymbol{X}$ and define

if

$$
\boldsymbol{x} \geqq * \boldsymbol{y}
$$

$$
f(\boldsymbol{x} ; \boldsymbol{\theta}) / f(\boldsymbol{x} ; \boldsymbol{\xi}) \geqq f(\boldsymbol{y} ; \boldsymbol{\theta}) / f(\boldsymbol{y} ; \boldsymbol{\xi}) \quad{ }^{\forall} \boldsymbol{\theta} \in \theta_{K_{0}},{ }^{\forall} \boldsymbol{\xi} \in \theta_{H_{0}} .
$$

The relation $\geqq_{*}$ induces a partial ordering on the sample space and a test statistic must be a generalization of $\geqq_{*}$. Let $A(\boldsymbol{x})=\left\{\boldsymbol{y} ; \boldsymbol{y} \geqq_{*} \boldsymbol{x}\right\}$ and $B(\boldsymbol{x})=\{\boldsymbol{y} ; \boldsymbol{x} \geqq * \boldsymbol{y}\}$. We

AMS 1970 Subject classifications. Primary $62 \mathrm{~F} 05$.

Key words and phrases. Normal populations, ordered alternative of means, ordered alternative of variances, partial ordering on sample space, test of homogeneity.

* Department of Statistics, Faculty of General Education, Osaka University. 
assume that the relation $\geqq_{*}$ is not trivial, i. e. $A(\boldsymbol{x}) \neq \phi$ or $B(\boldsymbol{x}) \neq \phi$ for some $\boldsymbol{x}$. The test statistics we shall consider are

and

$$
\begin{aligned}
& t_{1}=P\{A(\boldsymbol{X})\} / P\{A(\boldsymbol{X}) \cup B(\boldsymbol{X})\}, \\
& t_{2}=P\{A(\boldsymbol{X})\}, \\
& t_{3}=P\{A(\boldsymbol{X})\}-P\{B(\boldsymbol{X})\}
\end{aligned}
$$

$$
t_{\mathbf{4}}=-P\{B(\boldsymbol{X})\}
$$

where the probability $P$ is calculated under some adequate member of $\theta_{H_{0}}$. In many problems, it will be clear how to choose the element. Making use of each $t_{i}$ as a test statistic, the null hypothesis $H_{0}$ is rejected if $t_{i}$ is too small.

In Section 2 the problem $\left(H_{1}, K_{1}\right)$ is considered. This problem was studied by Bartholomew $[2,3,4,5]$ first and some generalizations were made by Chacko [6], Kudô [10], Nüesch [12], Shorack [17] and Perlman [13] and some nonparametric considerations were given by Jonckheere [10], Puri [14] and Tryon and Hettmansperger [18]. The book of Barlow et. al. gives a good summary. However, the parametric works of them are all based on the likelihood ratio test. Shirahata [15] studied bivariate one-sided problem which includes the problem $\left(H_{1}, K_{1}\right)$ for $k=3$ and known variance $\sigma^{2}$ from a new stand of view. It was shown that $t_{1}$ is better than the Bartholomew's $\bar{x}_{3}^{2}$ test by computer simulations. Hence it is expected that each $t_{i}$ is better than the likelihood ratio test for the unknown but common variance case. The statistic $t_{1}$ is also used to analyze a counted data in Shirahata [16]. A comparative study was given by Hirotsu [8].

In Section 3 the problem $\left(H_{2}, K_{2}\right)$ is considered. This problem may occur when we want to compare the accuracies of e.g. several instruments to measure. For the test of homogeneity of variances there are works of Fujino [7] and Vincent [19].

The explicit forms of $\geqq_{*}$ are given for the both problems. However, the distributions of $t_{i}$ 's are very complicated and to derive them are impossible analytically up to now even in the known variance case. The distributions vary according as $\boldsymbol{n}^{\prime}=\left(n_{1}, \cdots, n_{k}\right)$ varies and hence to determine cutoff points by simulations are also impractical. Therefore, in Section 4 we shall consider some artificial data, estimate their significances by simulations and compare the significances to the significances given by their competitors. It will be found that $t_{i}$ 's are good tests, especially $t_{3}$ and $t_{4}$ are very good. Hence we recommend to use $t_{3}$ or $t_{4}$ for the real data in both $\left(H_{1}, K_{1}\right)$ and $\left(H_{2}, K_{2}\right)$ by estimating the significance of the data empirically and then comparing it with the significance level.

\section{The problem of testing $H_{1}$ against $K_{1}$}

Put $\boldsymbol{\delta}^{\prime}=\left(\delta_{1}, \cdots, \delta_{k-1}\right)$ where $\delta_{i}=\theta_{i}-\theta_{i+1}$. Then $H_{1}$ and $K_{1}$ can be rewritten as $H_{1} ; \boldsymbol{\delta}=\mathbf{0}$ and $K_{1} ; \boldsymbol{\delta} \in D$ where $D=\left\{\boldsymbol{\delta} ; \delta_{i} \geqq 0\right.$ for $i=1, \cdots, k-1$ and $\left.\max _{i} \delta_{i}>0\right\}$. Thus, it is natural to consider tests based on

$$
\boldsymbol{T}^{\prime}=\left(T_{1}, \cdots, T_{k-1}\right)
$$


where

$$
\begin{aligned}
& T_{i}=a_{i}\left(\bar{X}_{i}-\bar{X}_{i+1}\right) / S, \\
& \bar{X}_{i}=\sum_{j=1}^{n_{i}} X_{i j} / n_{i}, \\
& a_{i}=\left\{m /\left(n_{i}^{-1}+n_{i+1}^{-1}\right)\right\}^{1 / 2}, \quad m=\sum_{i=1}^{k} n_{i}-k
\end{aligned}
$$

and

$$
S^{2}=\sum_{i=1}^{k} \sum_{j=1}^{n_{i}}\left(X_{i j}-\bar{X}_{i}\right)^{2}
$$

The statistic $\boldsymbol{T}$ is a multivariate central $t$-distribution under $H_{1}$ and is a noncentral $t$-distribution with nonnegative noncentrality parameters under $K_{1}$. From Johnson and Kotz [9] the density function of $\boldsymbol{T}$ is

$$
\begin{aligned}
f(\boldsymbol{t} ; \boldsymbol{\delta})= & \exp \left(-\boldsymbol{\delta}^{\prime} R^{-1} \boldsymbol{\delta} / 2\right)(m \pi)^{-(k-1) / 2}|R|^{-1 / 2} \Gamma^{-1}\left(\frac{m}{2}\right) \\
& \times\left(1+\frac{\boldsymbol{t}^{\prime} R^{-1} \boldsymbol{t}}{m}\right)^{-(m+k-1) / 2} h(\boldsymbol{t} ; \boldsymbol{\delta})
\end{aligned}
$$

where $R$ is a $(k-1) \times(k-1)$ matrix $\left(r_{i j}\right)$ with $r_{i i}=1, r_{i, i+1}=r_{i+1, i}=\rho_{i}$ for $\rho_{i}=-a_{i} a_{i+1} /$ $n_{i+1} m$ and $r_{i j}=0$ otherwise and where

$$
h(\boldsymbol{t} ; \boldsymbol{\delta})=\sum_{j=0}^{\infty} \frac{1}{j !} \Gamma\left(\frac{m+k+j}{2}\right)\left\{\left(\frac{2}{m+\boldsymbol{t}^{\prime} R^{-1} \boldsymbol{t}}\right)^{1 / 2} \boldsymbol{\delta}^{\prime} R^{-1} \boldsymbol{t}\right\}^{j} .
$$

Hence $f(\boldsymbol{t} ; \boldsymbol{\delta}) / f(\boldsymbol{t} ; \boldsymbol{0})$ is an increasing function of $h(\boldsymbol{t} ; \boldsymbol{\delta})$. Now the function

$$
\sum_{j=0}^{\infty} \frac{1}{j !} \Gamma\left(i+\frac{j}{2}\right) x^{j}
$$

is always positive for each $i>0, x \geqq 0$ and it is also found that it is an increasing function of $x$. Hence, considering the transformation

$$
\overline{\boldsymbol{T}}=R^{-1} \boldsymbol{T} /\left(m+\boldsymbol{T}^{\prime} R^{-1} \boldsymbol{T}\right)^{1 / 2}
$$

it follows that $\boldsymbol{t} \geqq_{*} \boldsymbol{s}$ if and only if $\boldsymbol{\delta}^{\prime} \overline{\boldsymbol{t}} \geqq \boldsymbol{\delta}^{\prime} \overline{\boldsymbol{s}}$ for each $\boldsymbol{\delta} \in D$. Therefore $\boldsymbol{t} \geqq_{*} \boldsymbol{s}$ if and only if

$$
\bar{t}_{i} \geqq \bar{s}_{i} \quad \text { for } \quad i=1, \cdots, k-1 .
$$

Now let us derive the density function of $\overline{\boldsymbol{T}}$ under $H_{1}$. It is easily shown that $0 \leqq \overline{\boldsymbol{T}}^{\prime} R \overline{\boldsymbol{T}} \leqq 1, \boldsymbol{T}^{\prime} R^{-1} \boldsymbol{T}=m \overline{\boldsymbol{T}}^{\prime} R \overline{\boldsymbol{T}} /\left(1-\overline{\boldsymbol{T}}^{\prime} R \overline{\boldsymbol{T}}\right)$. It is also shown that

$$
|\partial \boldsymbol{t} / \partial \overline{\boldsymbol{t}}|=\left\{\frac{m}{\left(1-\overline{\boldsymbol{t}}^{\prime} R \overline{\boldsymbol{t}}\right)^{3}}\right\}^{(k-1) / 2}|R| \times\left|\left(1-\overline{\boldsymbol{t}}^{\prime} R \overline{\boldsymbol{t}}\right) I_{k-1}+R \overline{\boldsymbol{t}} \overline{\boldsymbol{t}}^{\prime}\right|
$$

where $I_{k-1}$ is the $k-1$ dimensional unit matrix. Using a relation $\left|M+\boldsymbol{x} \boldsymbol{y}^{\prime}\right|=|M|(1$ $\left.+\boldsymbol{y}^{\prime} M^{-1} \boldsymbol{x}\right),(2.3)$ is

$$
|\partial \boldsymbol{t} / \partial \overline{\boldsymbol{t}}|=m^{(k-1) / 2}|R|\left(1-\overline{\boldsymbol{t}}^{\prime} R \overline{\boldsymbol{t}}\right)^{-(k+1) / 2} .
$$

Therefore the density function $f(\overline{\boldsymbol{t}})$ of $\overline{\boldsymbol{T}}$ under $H_{1}$ is given by 


$$
f(\overline{\boldsymbol{t}})= \begin{cases}\frac{\Gamma\left(\frac{m+k-1}{2}\right)}{\pi^{(k-1) / 2} \Gamma\left(\frac{m}{2}\right)}|R|^{1 / 2}\left(1-\overline{\boldsymbol{t}^{\prime}} R \overline{\boldsymbol{t}}\right)^{(m / 2)-1} & \text { for } \overline{\boldsymbol{t}^{\prime} R \overline{\boldsymbol{t}} \leqq 1} \\ 0 & \text { otherwise. }\end{cases}
$$

From (2.1), (2.2) and (2.4), $P\{A(\boldsymbol{T})\}$ and $P\{B(\boldsymbol{T})\}$ are calculated and hence $\boldsymbol{t}_{i}$ 's can be obtained. Note that if we consider further transformation $\overline{\bar{T}}=R^{1 / 2} \bar{T}$, then the density function $\bar{f}(\overline{\boldsymbol{t}})$ of $\overline{\overline{\boldsymbol{T}}}$ is

$$
\bar{f}(\overline{\overline{\boldsymbol{t}}})= \begin{cases}\frac{\Gamma\left(\frac{m+k-1}{2}\right)}{\pi^{(k-1) / 2} \Gamma\left(\frac{m}{2}\right)}\left(1-\overline{\left.\overline{\boldsymbol{t}}^{\prime} \boldsymbol{t}\right)^{(m / 2)-1}}\right. & \text { for } \overline{\overline{\boldsymbol{t}}^{\prime}} \overline{\overline{\boldsymbol{t}} \leqq 1} \\ 0 & \text { otherwise. }\end{cases}
$$

The formula (2.5) is very simple. It may be convenient to calculate $t_{i}$ 's from (2.5).

\section{The problem of testing $H_{2}$ against $K_{2}$}

Without loss of generality it can be assumed that each $\theta_{i}$ is unknown. Then it is natural to consider tests based on

where

$$
\boldsymbol{F}^{\prime}=\left(F_{1}, \cdots, F_{k-1}\right)
$$

and

$$
F_{i}=\left(n_{i+1}-1\right) S_{i}^{2} /\left(n_{i}-1\right) S_{i+1}^{2}
$$

$$
S_{i}^{2}=\sum_{j=1}^{n_{i}}\left(X_{i j}-\bar{X}_{i}\right)^{2} .
$$

The statistic $F_{i}$ is a $F$-distribution with degrees of freedom $\left(n_{i}-1, n_{i+1}-1\right)$ under $H_{2}$. Under $K_{2}$ it is distributed as $\sigma_{i}^{2} / \sigma_{i+1}^{2}$ multiplied by the above $F$-distribution and hence it is stochastically larger than the distribution under $H_{2}$.

After a simple calculation the natural $\log$ of the density function of $\boldsymbol{F}$ is given by

$$
\begin{aligned}
\log h\left(\boldsymbol{f} ; \sigma_{1}, \cdots, \sigma_{k}\right)= & c-\sum_{i=1}^{k}\left(n_{i}-1\right) \log \sigma_{i}+\sum_{i=1}^{k-1}\left(-1+\sum_{j=1}^{i} \frac{n_{j}-1}{2} \log f_{i}\right) \\
& -\frac{m}{2} \log \left(\frac{n_{k}-1}{\sigma_{k}^{2}}+\sum_{i=1}^{k-1} \frac{n_{i}-1}{\sigma_{i}^{2}} \prod_{j=i}^{k-1} f_{j}\right)
\end{aligned}
$$

where $c$ is a constant depending on $\boldsymbol{n}^{\prime}$ and $k$. Thus,

$$
\begin{aligned}
\log L\left(\boldsymbol{f} ; \sigma, \sigma_{1}, \cdots, \sigma_{k}\right) \\
=\log h\left(\boldsymbol{f} ; \sigma_{1}, \cdots, \sigma_{k}\right)-\log h(\boldsymbol{f} ; \sigma, \cdots, \boldsymbol{\sigma}) \\
=-\sum_{i=1}^{k}\left(n_{i}-1\right) \log \sigma_{i}+\frac{m}{2} \log \left\{n_{k}-1+\sum_{i=1}^{k-1}\left(n_{i}-1\right) \prod_{j=i}^{k-1} f_{j}\right\} \\
\quad-\frac{m}{2} \log \left(\frac{n_{k}-1}{\sigma_{k}^{2}}+\sum_{i=1}^{k-1} \frac{n_{i}-1}{\sigma_{i}^{2}} \prod_{j=i}^{k-1} f_{j}\right) .
\end{aligned}
$$


Hence, considering $\overline{\boldsymbol{F}}^{\prime}=\left(\bar{F}_{1}, \cdots, \bar{F}_{k-1}\right)$ where

$$
\bar{F}_{i}=\frac{n_{i}-1}{n_{k}-1} \prod_{j=i}^{k-1} F_{j},
$$

the necessary and sufficient condition of

$$
L\left(\boldsymbol{f} ; \sigma, \sigma_{1}, \cdots, \sigma_{k}\right) \geqq L\left(\boldsymbol{g} ; \sigma, \sigma_{1}, \cdots, \sigma_{k}\right)
$$

for each $\sigma_{1} \geqq \cdots \geqq \sigma_{k}>0$ is

$$
\begin{aligned}
\sum_{i=1}^{k-1}\left(\bar{f}_{i}-\bar{g}_{i}\right) & \geqq \sum_{i=1}^{k-1} \frac{\sigma_{k}^{2}}{\sigma_{i}^{2}}\left\{\bar{f}_{i}-\bar{g}_{i}+\sum_{j=1}^{k-1}\left(\bar{f}_{i} \bar{g}_{j}-\bar{f}_{j} \bar{g}_{i}\right)\right\} \\
& =\sum_{i=1}^{k-1} \frac{\sigma_{k}^{2}}{\sigma_{i}^{2}} a_{i} \text { (say). }
\end{aligned}
$$

Since the inequality (3.2) is

$$
\sum_{i=1}^{k-1}\left(\lambda_{i}-\lambda_{i+1}\right) \sum_{j=1}^{i} a_{i} \leqq 0
$$

where $\lambda_{i}=\sigma_{k}^{2} / \sigma_{i}^{2}$, (3.2) holds if and only if $\sum_{j=1}^{i} a_{j} \geqq 0$ for $i=1, \cdots, k-1$ and hence $\boldsymbol{f} \geqq_{*} \boldsymbol{g}$ if and only if

$$
\sum_{j=1}^{i} \bar{f}_{j} /\left(1+\sum_{j=1}^{k-1} \bar{f}_{j}\right) \geqq \sum_{j=1}^{i} \bar{g}_{j} /\left(1+\sum_{j=1}^{k-1} \bar{g}_{j}\right) \quad \text { for } \quad i=1, \cdots, k-1 .
$$

Put $\overline{\overline{\boldsymbol{F}}^{\prime}}=\left(\overline{\bar{F}}_{1}, \cdots, \overline{\bar{F}}_{k-1}\right)$ where

$$
\begin{aligned}
\bar{F}_{i} & =\sum_{j=1}^{i} \bar{F}_{j} /\left(1+\sum_{j=1}^{k-1} \bar{F}_{j}\right) \\
& =\sum_{j=1}^{i} S_{j}^{2} / \sum_{j=1}^{k} S_{j}^{2} .
\end{aligned}
$$

Then the density function of $\overline{\overline{\boldsymbol{F}}}$ is

$$
g\left(w_{1}, \cdots, w_{k-1}\right)=\frac{\Gamma\left(\frac{m}{2}\right)}{\prod_{i=1}^{k} \Gamma\left(\frac{n_{i}-1}{2}\right)^{k}} \prod_{i=1}^{k}\left(w_{i}-w_{i-1}\right)^{\left(n_{i}-3\right) / 2}
$$

for $w_{0}=0<w_{1}<\cdots w_{k-1}<1=w_{k}$ and $g\left(w_{1}, \cdots, w_{k-1}\right)=0$ otherwise.

From (3.1), (3.3), (3.4) and (3.5), $P\{A(\boldsymbol{F})\}$ and $P\{B(\boldsymbol{F})\}$ can be calculated.

\section{Some numerical results}

From the results in Sections 2 and 3, we can calculate four statistics $t_{1}, t_{2}, t_{3}$ and $t_{4}$. But, the distributions of them depend on $\boldsymbol{n}^{\prime}=\left(n_{1}, \cdots, n_{k}\right)$ and further it is impossible to obtain the distributions even for a fixed $\boldsymbol{n}$. However, to perform a significance test, it is enough to know the significance of the given data. The power of the $t_{i}$ test can be guessed by estimating the significances of several artificial data. 
Hence we estimate the significances of some artificial data by simulations and then compare with the significances given by the competitors. It will be found that our statistics are powerful.

We consider only the case $k=3$ and $\boldsymbol{n}^{\prime}=(7,5,3),(5,5,5)$ and $(3,5,7)$. The artificial data to be investigated are $\left(\bar{X}_{1}-\bar{X}_{2}, \bar{X}_{2}-\bar{X}_{3}, S^{2}\right)=(0.8,0,12),(0.4,0.4,12)$ and $(0,0.8,12)$ for the problem $\left(H_{1}, K_{1}\right)$. They correspond to the cases $\theta_{1}>\theta_{2}=\theta_{3}, \theta_{1}-\theta_{2}$ $=\theta_{2}-\theta_{3}>0$ and $\theta_{1}=\theta_{2}>\theta_{3}$, respectively. For the problem $\left(H_{2}, K_{2}\right)$ we consider

$$
\left(\frac{S_{1}^{2}}{n_{1}-1}, \frac{S_{2}^{2}}{n_{2}-1}, \frac{S_{3}^{2}}{n_{3}-1}\right)=(3,3,1),(3, \sqrt{3}, 1)
$$

and $(3,1,1)$. They correspond to $\sigma_{1}=\sigma_{2}>\sigma_{3}, \sigma_{1} / \sigma_{2}=\sigma_{2} / \sigma_{3}>1$ and $\sigma_{1}>\sigma_{2}=\sigma_{3}$ respectively. These data are analyzed except the cases $\boldsymbol{n}^{\prime}=(3,5,7)$ and $\boldsymbol{n}^{\prime}=(5,5,5)$ with $\left(\bar{X}_{1}-\bar{X}_{2}, \bar{X}_{2}-\bar{X}_{3}, S^{2}\right)=(0,0.8,12)$ for the problem $\left(H_{1}, K_{1}\right)$. There exceptions are due to the fact that for the problem $\left(H_{1}, K_{1}\right)$ we can show as in Shirahata [15] that the power for the alternative $\left(\delta_{1}, \delta_{2}\right)$ with $\left(n_{1}, n_{2}, n_{3}\right)$ is equal to the power for the alternative $\left(\delta_{2}, \delta_{1}\right)$ with $\left(n_{3}, n_{2}, n_{1}\right)$.

For five cases in the problem $\left(H_{1}, K_{1}\right)$ we simulated 100 times in $\{A(\boldsymbol{X}) \cup B(\boldsymbol{X})\}^{c}$

Table 1. Empirical significances of artificial data in the problem $\left(H_{1}, K_{1}\right)$.

\begin{tabular}{|c|c|c|c|c|c|c|c|c|c|}
\hline $\boldsymbol{n}^{\prime}$ & $\left(\bar{X}_{1}-\bar{X}_{2}{ }^{\mathrm{d}}\right.$ & $\left.\bar{X}_{2}-\bar{X}_{3}, S^{2}\right)$ & $t_{1}$ & $t_{2}$ & $t_{3}$ & $t_{4}$ & $\overline{F_{3}}$ & $\overrightarrow{E^{2}}$ & $t$ \\
\hline \multirow{3}{*}{$(7,5,3)$} & $(0.8$ & $0,12)$ & 0.095 & 0.105 & 0.091 & 0.091 & 0.205 & 0.124 & 0.097 \\
\hline & $(0.4$ & $0.4,12)$ & 0.068 & 0.093 & 0.052 & 0.050 & 0.294 & 0.205 & 0.127 \\
\hline & $(0$ & $0.8,12)$ & 0.151 & 0.164 & 0.135 & 0.140 & 0.282 & 0.194 & 0.165 \\
\hline \multirow{2}{*}{$(5,5,5)$} & $(0.8$ & $0,12)$ & 0,116 & 0.130 & 0.112 & 0.116 & 0.222 & 0.139 & 0.115 \\
\hline & $(0.4$ & $0.4,12)$ & 0.136 & 0.150 & 0.098 & 0.092 & 0.272 & 0.184 & 0.115 \\
\hline
\end{tabular}

Table 2. Empirical significances of artificial data in the problem $\left(H_{2}, K_{2}\right)$.

\begin{tabular}{|c|c|c|c|c|c|c|c|c|c|}
\hline$n^{\prime}$ & $\left(\frac{S_{1}^{2}}{n_{1}-1}\right.$ & $\begin{array}{c}\text { data } \\
S_{2}^{2} \\
n_{2}-1\end{array}$ & $\left.\frac{S_{3}^{2}}{n_{3}-1}\right)$ & $t_{1}$ & $t_{2}$ & $t_{3}$ & $t_{4}$ & $B_{a}$ & $L R$ \\
\hline \multirow{3}{*}{$(7,5,3)$} & & $(3, \quad 3$, & & 0.297 & 0.339 & 0.274 & 0.253 & 0.716 & 0.380 \\
\hline & & $(3, \sqrt{ } 3$ & & 0.258 & 0.280 & 0.198 & 0.179 & 0.664 & 0.360 \\
\hline & & $(3, \quad 1$, & & 0.185 & 0.210 & 0.161 & 0.163 & 0.437 & 0.199 \\
\hline \multirow{3}{*}{$(5,5,5)$} & & $(3, \quad 3$ & & 0.186 & 0.208 & 0.173 & 0.163 & 0.542 & $0 \cdot 192$ \\
\hline & & $(3, \sqrt{ } 3$ & & 0.160 & 0.191 & 0.122 & 0.112 & 0.573 & 0.249 \\
\hline & & $(3,1$, & & 0.138 & 0.154 & 0.108 & 0.107 & 0.454 & 0.174 \\
\hline \multirow{3}{*}{$(3,5,7)$} & & $(3, \quad 3$, & & 0.135 & 0.140 & 0.116 & 0.117 & 0.479 & 0.134 \\
\hline & & $(3, \sqrt{ } 3$ & & 0.141 & 0.173 & 0.104 & 0.095 & 0.636 & 0.213 \\
\hline & & $(3, \quad 1$, & & 0.172 & 0.188 & 0.129 & 0.118 & 0.588 & 0.169 \\
\hline
\end{tabular}


and for nine cases in the problem $\left(H_{2}, K_{2}\right)$, we simulated 150 times in $\{A(\boldsymbol{X}) \cup B(\boldsymbol{X})\}^{c}$. Table 1 and 2 gives

$$
P(A)+\{1-P(A)-P(B)\} r_{2} / r_{1}
$$

where we denote by $P(A)$ and $P(B)$ the probabilities of the sets $A(\boldsymbol{x})$ and $B(\boldsymbol{x})$ of our artificial data. Here $r_{1}=100$ in the problem $\left(H_{1}, K_{1}\right), r_{1}=150$ in the problem $\left(H_{2}, K_{2}\right)$ and $r_{2}$ is the number of times of random numbers more significant than the artificial data. The formula (4.1) gives an estimate of the significance of the data. Table 1 also includes theoretical significances of Bartholomew's $\bar{F}_{3}, \vec{E}^{2}$ and usual $t$-statistic

$$
t=-m^{1 / 2} \sum_{i=1}^{k} i n_{i}\left(\bar{X}_{i}-\bar{X}\right) / S\left\{\sum_{i=1}^{k} n_{i}\left(i-\sum_{j=1}^{k} j \frac{n_{j}}{n}\right)^{2}\right\}^{1 / 2}
$$

where $\bar{X}=n^{-1} \sum_{i=1}^{k} \sum_{j=1}^{n_{i}} X_{i j}$ and $n=\sum_{i=1}^{k} n_{i}$. The statistic $t$ has $t$-distribution with degree of freedom $m$. Table 2 includes the empirical significances given by Bartlett's test

$$
B_{a}=m\left\{\log \left(m^{-1} \sum_{i=1}^{k} S_{i}^{2}\right)-m^{-1} \sum_{i=1}^{k}\left(n_{i}-1\right) \log \frac{S_{i}^{2}}{n_{i}-1}\right\}
$$

and the likelihood ratio test $L R$ based on $\left(S_{1}^{2}, S_{2}^{2}, S_{3}^{2}\right)$ where

$$
L R=\left\{\begin{array}{c}
m \log \frac{S_{1}^{2}+S_{2}^{2}+S_{3}^{2}}{m}-\sum_{i=1}^{3}\left(n_{i}-1\right) \log \frac{S_{i}^{2}}{n_{i}-1}, \\
\text { for } \frac{S_{1}^{2}}{n_{1}-1} \geqq \frac{S_{2}^{2}}{n_{2}-1} \geqq \frac{S_{3}^{2}}{n_{3}-1}, \\
m \log \frac{S_{1}^{2}+S_{2}^{2}+S_{3}^{2}}{m}-\left(n_{1}-1\right) \log \frac{S_{1}^{2}}{n_{1}-1}-\left(n_{2}+n_{3}-2\right) \log \frac{S_{2}^{2}+S_{3}^{2}}{n_{2}+n_{3}-2}, \\
\text { for } \frac{S_{1}^{2}}{n_{1}-1} \geqq \frac{S_{3}^{2}}{n_{3}-1} \geqq \frac{S_{2}^{2}}{n_{2}-1} \\
m \log \frac{S_{1}^{2}+S_{2}^{2}+S_{3}^{2}}{m}-\left(n_{1}+n_{2}-2\right) \log \frac{S_{1}^{2}+S_{2}^{2}}{n_{1}+n_{2}-2}-\left(n_{3}-1\right) \log \frac{S_{3}^{2}}{n_{3}-1} \\
\text { for } \frac{S_{2}^{2}}{n_{2}-1} \geqq \frac{S_{1}^{2}}{n_{1}-1} \geqq \frac{S_{3}^{2}}{n_{3}-1}, \\
\text { otherwise. }
\end{array},\right.
$$

For $B_{a}$ and $L R$, the calculations were done 1000 times. In general the smaller significance a test gives, the more powerful the test is. Therefore, from the Tables we can conclude that tests based on $t_{3}$ and $t_{4}$ are very powerful in the both problems.

REMARK 4.1. It will be worth mentioned that the significance given by $t_{i}$ is larger than $P(A)$ and is smaller than $1-P(B)$. Therefore if $1-P(B)$ is smaller than the significance level or $P(A)$ is larger than it, we need not simulate. Any test which is an extension of $\geqq_{*}$ has this property. But, because Bartholomew's $\bar{F}_{k}$ and $\bar{E}^{2}$ are not based on $\boldsymbol{T}$ they do not necessarily have this property in our formulation.

REMARK 4.2. It is very difficult to calculate $t_{i}$ 's for large $n_{i}$ 's and $k$. Considering, however, their power properties, they are useful at least for $k=3$. 


\section{References}

[1] Barlow, R.E., Bartholomew, D. J., Bremner, J.M. and Brunk, H.D.: Statistical Inference under Order Restrictions. (1972). John Wiley and Sons, New York.

[2] Bartholomew, D.J.: A test of homogeneity for ordered alternatives. Biometrika, 46 (1959), 36-48.

[3] Bartholomew, D.J.: A test of homogeneity for ordered alteranatives II. Biometrika, 46 (1959), 328-335.

[4] BARTHOLOMEw, D. J.: A test of homogeneity of means under restricted alternatives. (with discussion). J. R. Statist. Soc. B, 23 (1961), 239-281.

[5] Bartholomew, D. J.: Ordered tests in the analysis of variance. Biometrika, 48 (1961), 325-332.

[6] Снаско, V.J.: Testing homogeneity against ordered alternatives. Ann. Math. Statist., 34 (1963), 945-956.

[7] Fujsio, K.: A likelihood ratio test for homogeneity of variances against ordered alter. natives. Jap. J. Appl. Statist., 6 (1977), 3-12. (in Japanese)

[8] Hirotsu, C.: The Analysis of Variance. (1976), Kyoiku Shuppan, Tokyo. (in Japanese)

[9] Johnson, N.L. and Kotz, S.: Distributions in Statistics: Continuous Multivariate Distributions. (1972), John Wiley and Sons, New York.

[10] JonckheEre, A.R.: A distribution-free $k$-sample test against ordered alternatives. Biometrika 41 (1954), 133-145.

[11] Kudô, A.: A multivariate analogue of the one-sided test. Biometrika, 50 (1963), 403-418.

[12] NUESCH, P.E.: On the problem of testing locations in multivariate populations for restricted alternatives. Ann. Math. Statist., 37 (1966), 113-119.

[13] Perlman, M.D.: One-sided testing problems in multivariate analysis. Ann. Math. Statist., 40 (1969), 549-567.

[14] PURI, M.L.: Some distribution-free k-sample rank tests of homogeneity against ordered alternatives. Comm. Pure Appl. Math., 18 (1965), 51-63.

[15] Shirahata, S.: An approach to the one-sided test in the bivariate normal population. Biometrika, 65 (1978), 61-67.

[16] Shirahata, S.: Exact test on percentages in a two-way table. Biometrics, to appear.

[17] SHORACK, G.R.: Testing against ordered alternatives in model I analysis of variance; normal theory and nonparametric. Ann. Math. Statist., 38 (1967), 1740-1753.

[18] Tryon, P.V. and Hettmansperger, T.P.: A class of non-parametric tests for homogeneity against ordered alternatives. Ann. Statist., 1 (1973), 1061-1070.

[19] Vincent, S.E.: A test of homogeneity for ordered variances. J.R. Statist. Soc. B, 23 (1961), 195-206. 